\title{
دراسة حصرية لأمواع الارضة في محافظة عدن - اليمن
}

ناصر سعيد بافاضل 1، سعيد عبد الله باعنقد 20

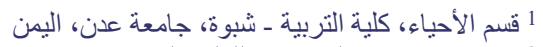

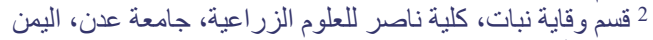

$$
\begin{aligned}
& 3 \text { قسم الأحياء، كلية التربية ــ عدن، جامعة عدن، اليمن الئن }
\end{aligned}
$$

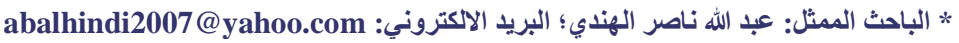

استلم في: 01 أغسطس 2020 / قبل في: 24 أكتوبر 2020 / نثر في: 30 ديسمبر 2020

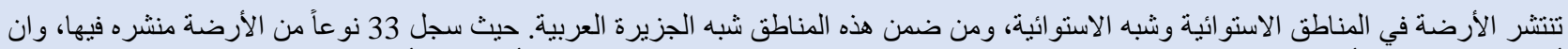

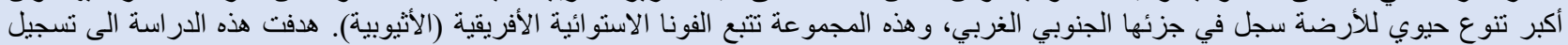

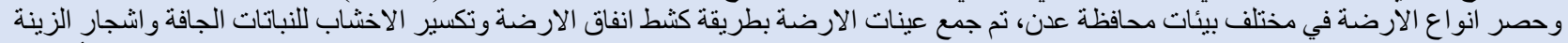

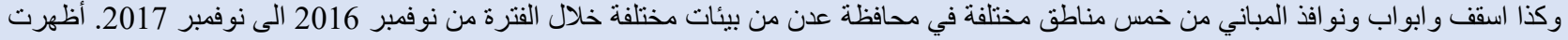

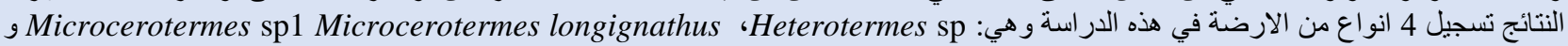

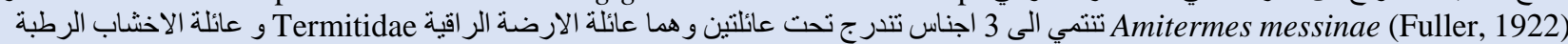

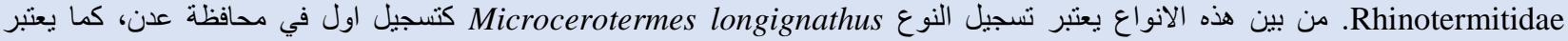
Heterotermes sp

الكلمات المفتاحية: الارضة، انتثار، النوع، محافظة عدن، اليمن.

أشار [15] إلى إن العدد المسجل من أنواع الأرضة في اليمن قد وصل إلى الكي

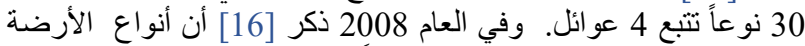

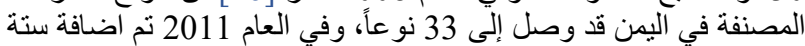

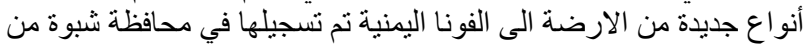

قبل [17] و يصبح عدد انواع الارغة الارضة التي سجلت في اليمن 39 نو عا.

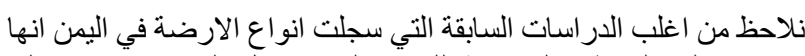

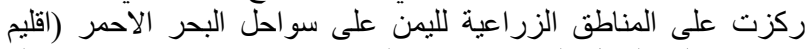

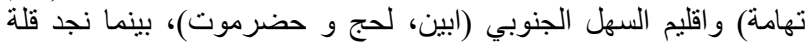

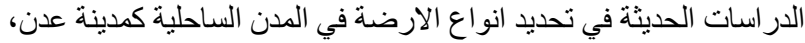

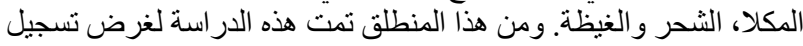

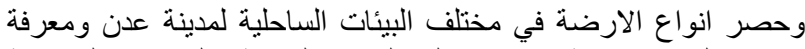

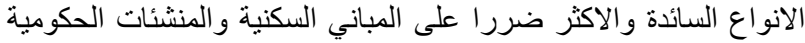
و الغطاء النباتي.

$$
\text { 2.1 } 2.1
$$

تم جمع عينات الارضة من خمس مناطق مختلفة البيئات في محافظة عدن القن

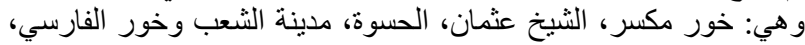

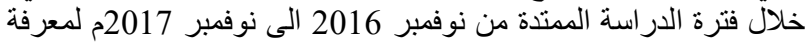

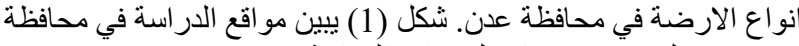
عدن و جدول (1) يبين تفاصيل مواقع الدراسة.

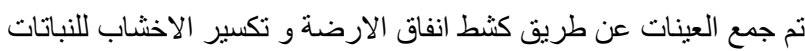
الجافة (الدمس , Conocarpus lancifolus المريمرة Azadrrachta

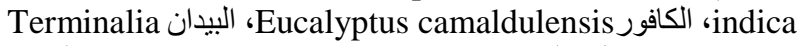
catappa ونو Lawsonia inermis L) ، (Lantan camara L. ونو افذ المباني بحسب طريقة [17]. تم وضع العينات المجمعة في أوعية

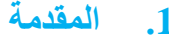

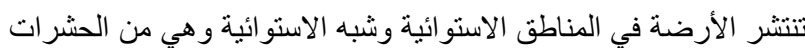

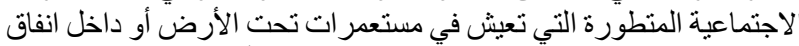

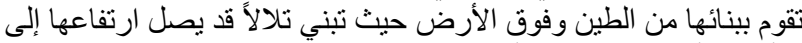

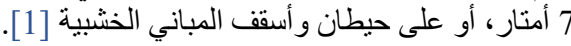

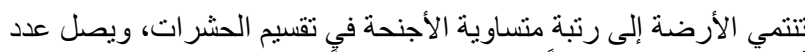
أنو اعها إلى 2700 نو عاً تتنمي ل 282 جنساً، وتتبع سبع عو ائل [2 و 33].

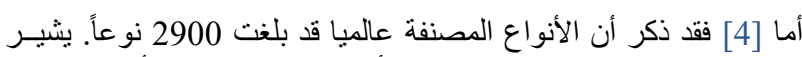

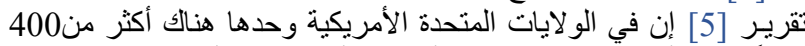

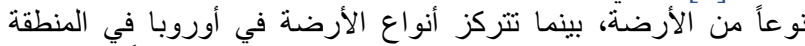

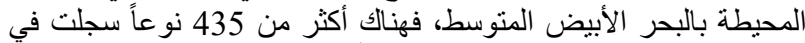

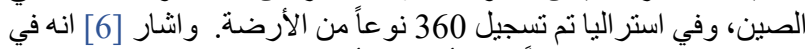

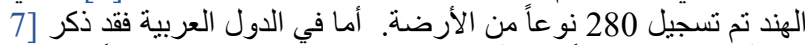

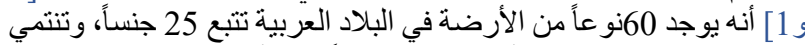

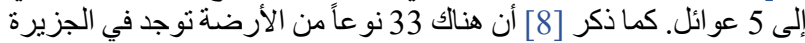

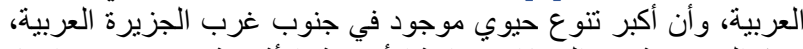

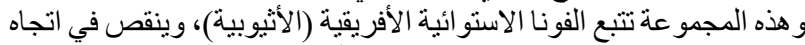

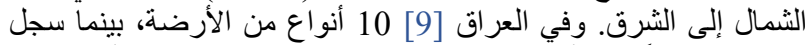

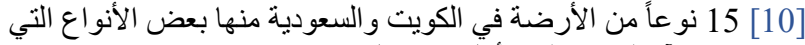

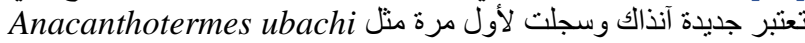

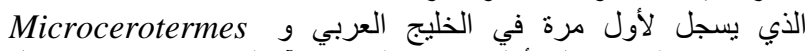
parvenus

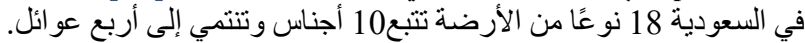

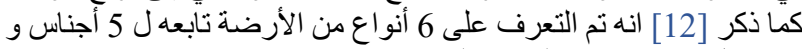
3 عو ائل في الإمار ات العربية المتحدة.

ازداد عدد أنواع الأرضة المصنفة في اليمن في ثمانينيات القرن المنصرم، إنما، فقد سجل [13] 16 نو عاً من الارضة الردة، اما [14] فقد سجل 24 نوعاً، بينما 
لغرض تصنيف العينات التي N1H 3A1- Dr. Timothy G. Myles) و اجهناصعوبة في تصنيفها وكذا التأكد من تصنيف العينات التي تم تصنيفها.

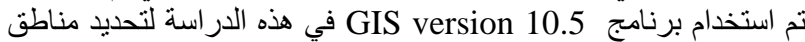
الدر اسة وكذا انتشار انواع الار الارضة في هذه المناطق، كما تم احصائية حسابية لمعرفة نسب نو اجد الانواع. النواع.

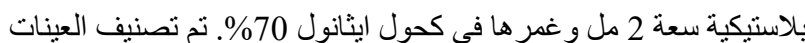

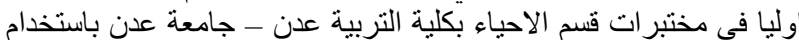

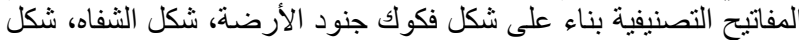

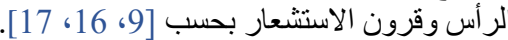

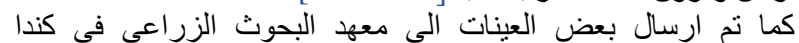
(Termite Control Officer- Carden St., Ontario, Canada

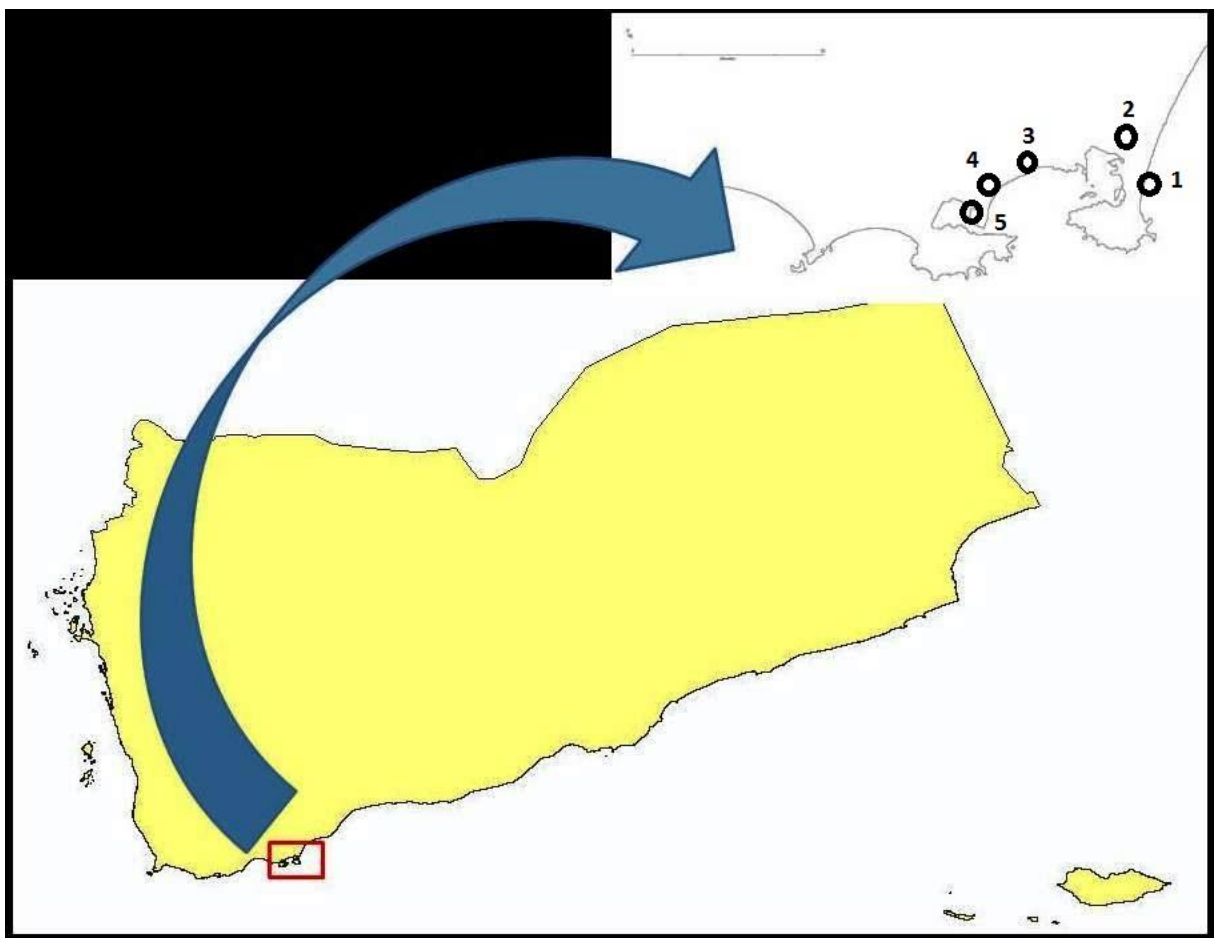

شكل 1: يوضح مناطق جمع عينات الارضة في محافظة عدن، اليمن.

وفي مختلف الاماكن من مبان سكنية واشجار، بينما بعتبر النوع

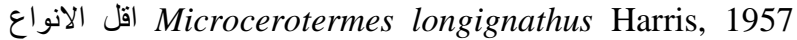

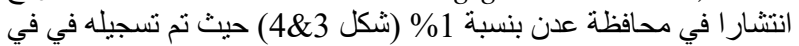

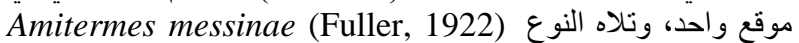

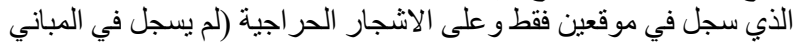

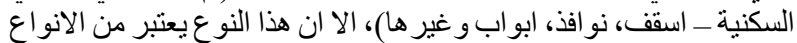

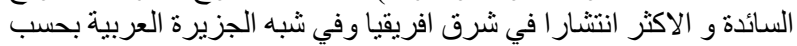

تم تسجيل 11 نو عا من الارضة في مدينة عدن و المناطق المجاورة لها من قبل [18]، حيث ذكر ان النوع Microcerotermes diversus هو الأوف

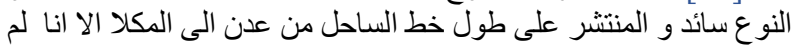
نجده خلال فترة الدر اسة في محافظة طئ عدن.

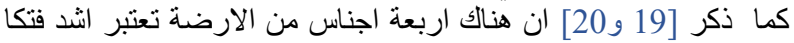

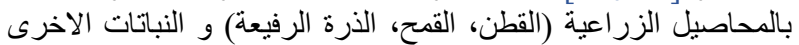

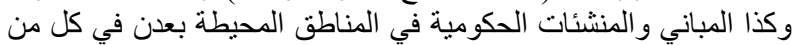
ابين ولحج، و هذه الاجناس هي:
3. النتائج والمناقشة

تم تسجيل 4 انواع من الارضة وهي: Heterotermes sp، Microcerotermes Microcerotermes longignathus Harris, Amitermes messinae (Fuller, 1922) و 1958 اجناس تتدرج تحت عائلتين وهما عائلة الارضة الراقية Termtidae

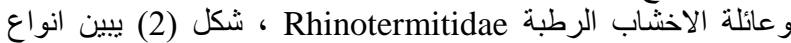

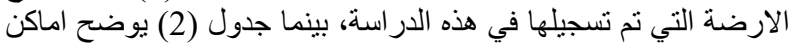

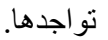

Microcerotermes longignathus يعتبر تسجيل انتشار النوع ري في هذه الدراسة تسجيل اول في محافظة عدن في الموقع

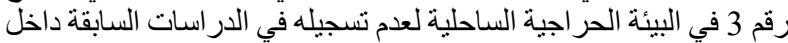

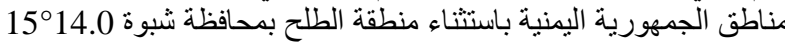
(47²28.190 E \&N)

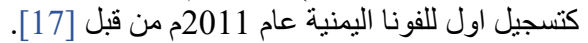

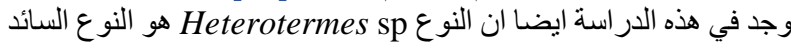
في محافظة عدن بنسبة 64\% حيث تم تسجيله في جميع مو اقع الدر اسة النة

جدول 1: يبين مو اقع جمع عينات الارضة في محافظة عدن:

\begin{tabular}{|c|c|c|c|c|c|}
\hline اماكن الجمع & 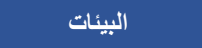 & الارتفاع & الاحداثيات & اسم الموقع & الرق \\
\hline حدائق، مباني & حر اجية ساحلية & 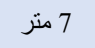 & $12^{\circ} 48.705 \mathrm{~N} 45^{\circ} 02.400 \mathrm{E}$ & خور مكسر & 1 \\
\hline حدائق، مشاتل & حر اجية طينية رملية & 14 متر & $12^{\circ} 52.650 \mathrm{~N} 44^{\circ} 59.049 \mathrm{E}$ & الثيخ عثمان & 2 \\
\hline حدائق، اشجار حراجية & حراجية ساحلية & 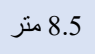 & $12^{\circ} 50.090 \mathrm{~N} 44^{\circ} 57.481 \mathrm{E}$ & الحسوة & 3 \\
\hline مباني، حدائق & حر اجية طينية رملية & 20 منر & $12^{\circ} 50.186 \mathrm{~N} 44^{\circ} 55.002 \mathrm{E}$ & مدينة الثعب & 4 \\
\hline مباني، حدائق & طبية ساحلية & 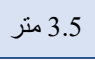 & $12^{\circ} 46.563 \mathrm{~N} \quad 44^{\circ} 53.438 \mathrm{E}$ & خور الفارسي & 5 \\
\hline
\end{tabular}


جدول 2: يبين تواجد انواع الارضة في مو اقع الدر اسة بمحافظة عدن في هذه الدراسة الحالية ( \ تسجيل اول، + تو اجد النوع، - عدم تواجد النوع):

\begin{tabular}{|c|c|c|c|c|c|c|c|c|c|}
\hline \multicolumn{2}{|c|}{ الماكن التواجد } & \multirow{2}{*}{ موقع 5} & \multirow{2}{*}{ هوقع 4} & \multirow{2}{*}{ موقع 3} & \multirow{2}{*}{ موقع 2} & \multirow{2}{*}{ موقع 1} & \multirow{2}{*}{ 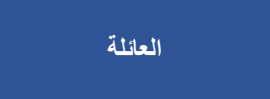 } & \multirow{2}{*}{ نوع الارضة } & \multirow{2}{*}{ a } \\
\hline اشجار & 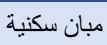 & & & & & & & & \\
\hline+ & + & + & + & + & + & + & عائلة ارضة الاخشاب الرطبة & Heterotermes sp & 1 \\
\hline+ & + & - & - & + & - & - & عائلة الارضة الر اقية & Microcerotermes longignathus $\boldsymbol{\Delta}$ & 2 \\
\hline+ & - & - & + & + & + & - & $\begin{array}{l}\text { Termitidae } \\
\text { تحت عائلة }\end{array}$ & Microcerotermes $\mathrm{sp}$ & 3 \\
\hline+ & - & - & + & - & - & + & Termitinae & Amitermes messinae & 4 \\
\hline
\end{tabular}

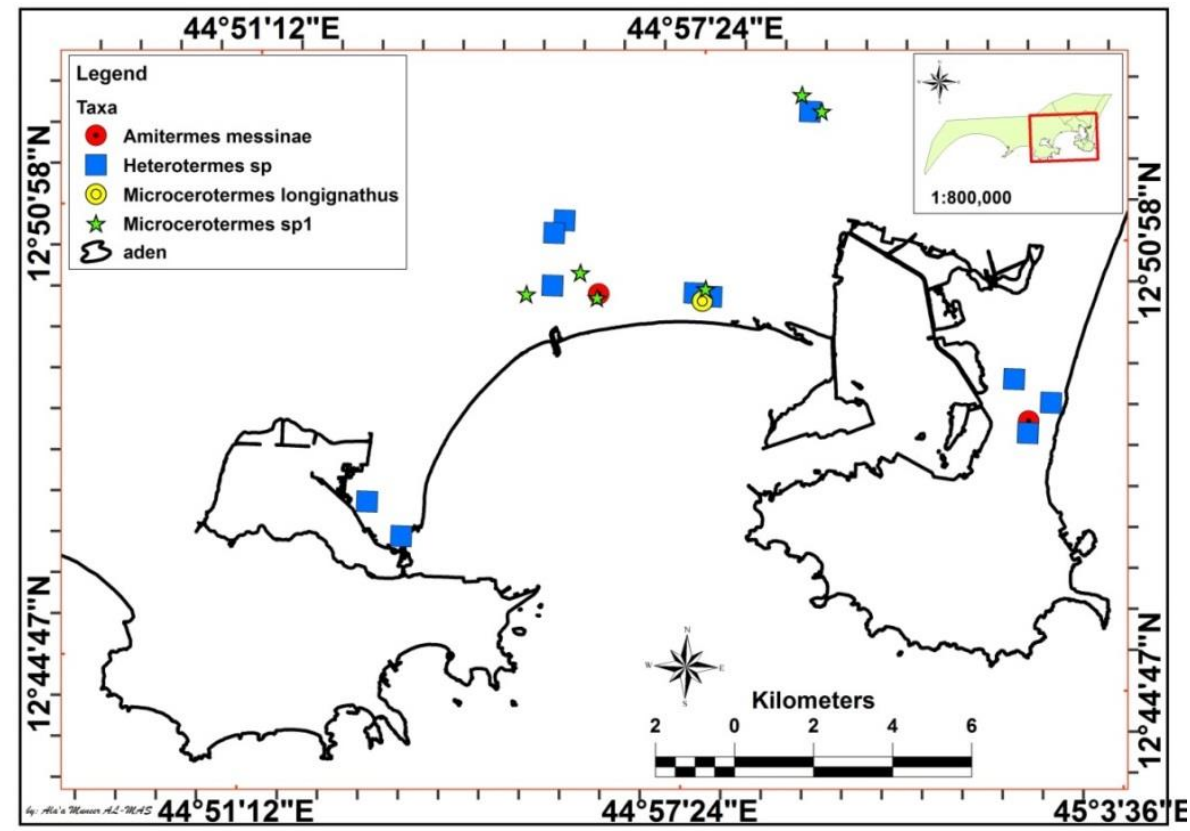

شكل 3: يبين مو اقع تو اجد الانواع المتحصل عليها في مناطق الدراسة.

تم تسجيل 8 انو اع منوطنة في شبة الجزيرة العربية تابعة لخمس عائلات من

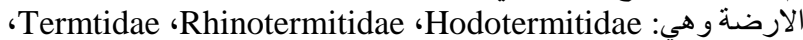
Nermitinae

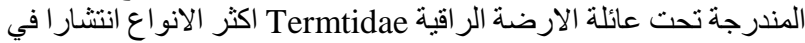

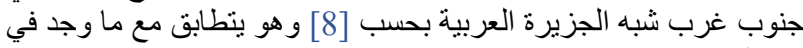
هذه الدر اسة.

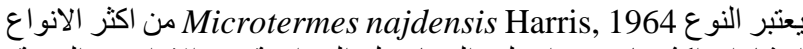

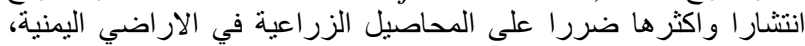

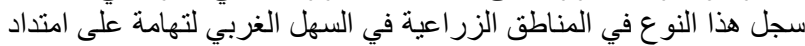

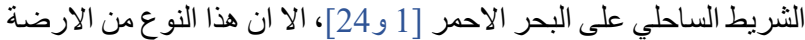

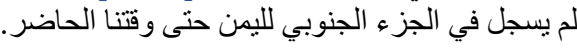

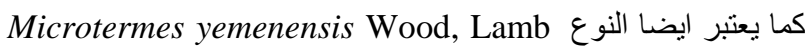

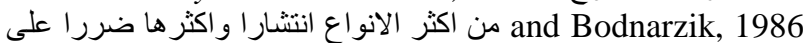

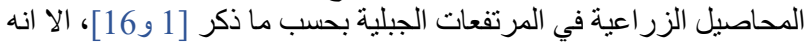

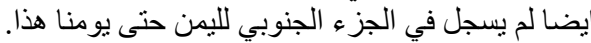

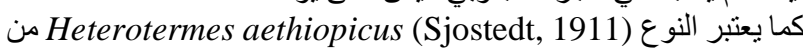

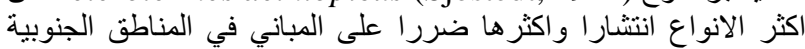

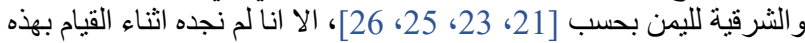
الدراسة، الذي وجد في هذه الدراسة هو

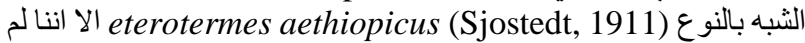
نستطع الجزم بانه نفس النوع، نحتاج للمزيد من الدراسات التصنيفية

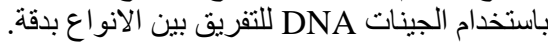

Amitermes sp microtermes spp , 'Heterotermes spp : .Microcerotermes spp, Epicalotermes spp

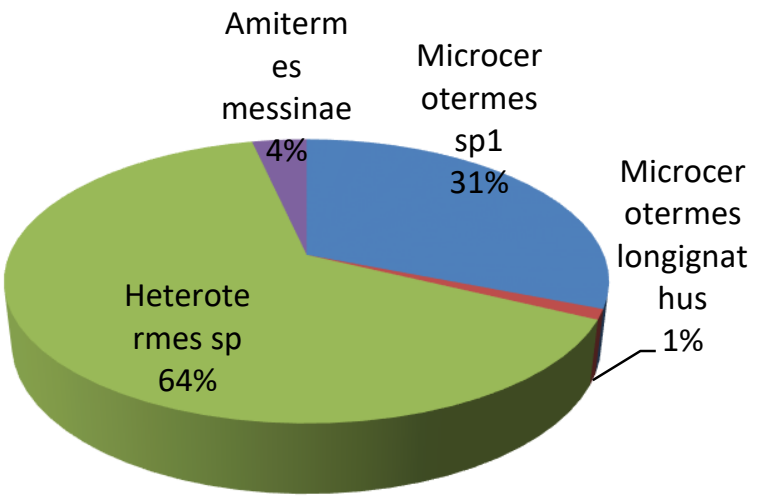

شكل 4: يبين النسبة المئوية لتو اجد كل نوع في منطقة الدراسة.

بينما سجل [21] في دلتا ابين 7 انو اع من الارضة تندرج تحت 7 اجناس،

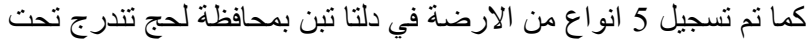

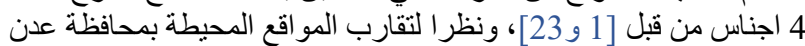

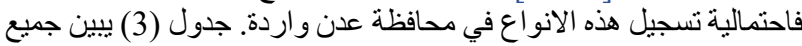

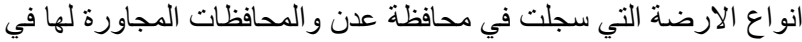
هذه الدر اسة و الار أسات السابقة. 
جدول 3: يبين تو اجد انواع الارضة في محافظة عدن و المناطق المجاورة لها في هذه الدراسة و الدراسات السابقة (* الدراسة الحالية، *1 [21]، *23] [23]، (ن)

\begin{tabular}{|c|c|c|c|c|c|c|c|}
\hline $5 \%$ & $4 \%$ & $3 *$ & $2 \%$ & $1 \%$ & * & نوع الارضة & f \\
\hline+ & + & - & - & - & + & Heterotermes $\mathrm{sp}$ & 1 \\
\hline- & - & - & - & - & $\boldsymbol{\Delta}$ & Microcerotermes longignathus & 2 \\
\hline+ & + & - & - & - & + & Amitermes messinae & 3 \\
\hline+ & + & + & + & + & - & Heterotermes aethiopicus & 4 \\
\hline+ & - & - & - & - & - & Macrotermes subhyalinus & 5 \\
\hline+ & + & + & + & + & - & Microcerotermes diversus & 6 \\
\hline+ & + & + & - & + & - & Psammotermes hybostoma & 7 \\
\hline+ & + & - & - & - & - & Amitermes stephensoni & 8 \\
\hline+ & + & + & - & - & + & Microcerotermes $s p$ & 9 \\
\hline+ & + & - & - & - & - & Epicalotermes aethiopicus & 10 \\
\hline- & - & - & - & + & - & Anacanthoterme ochraceous & 11 \\
\hline+ & + & - & - & + & - & Reticulitermes sp & 12 \\
\hline- & - & - & - & + & - & Kaltermes sp & 13 \\
\hline+ & + & + & - & + & - & Amitermes loennbergianus & 14 \\
\hline
\end{tabular}

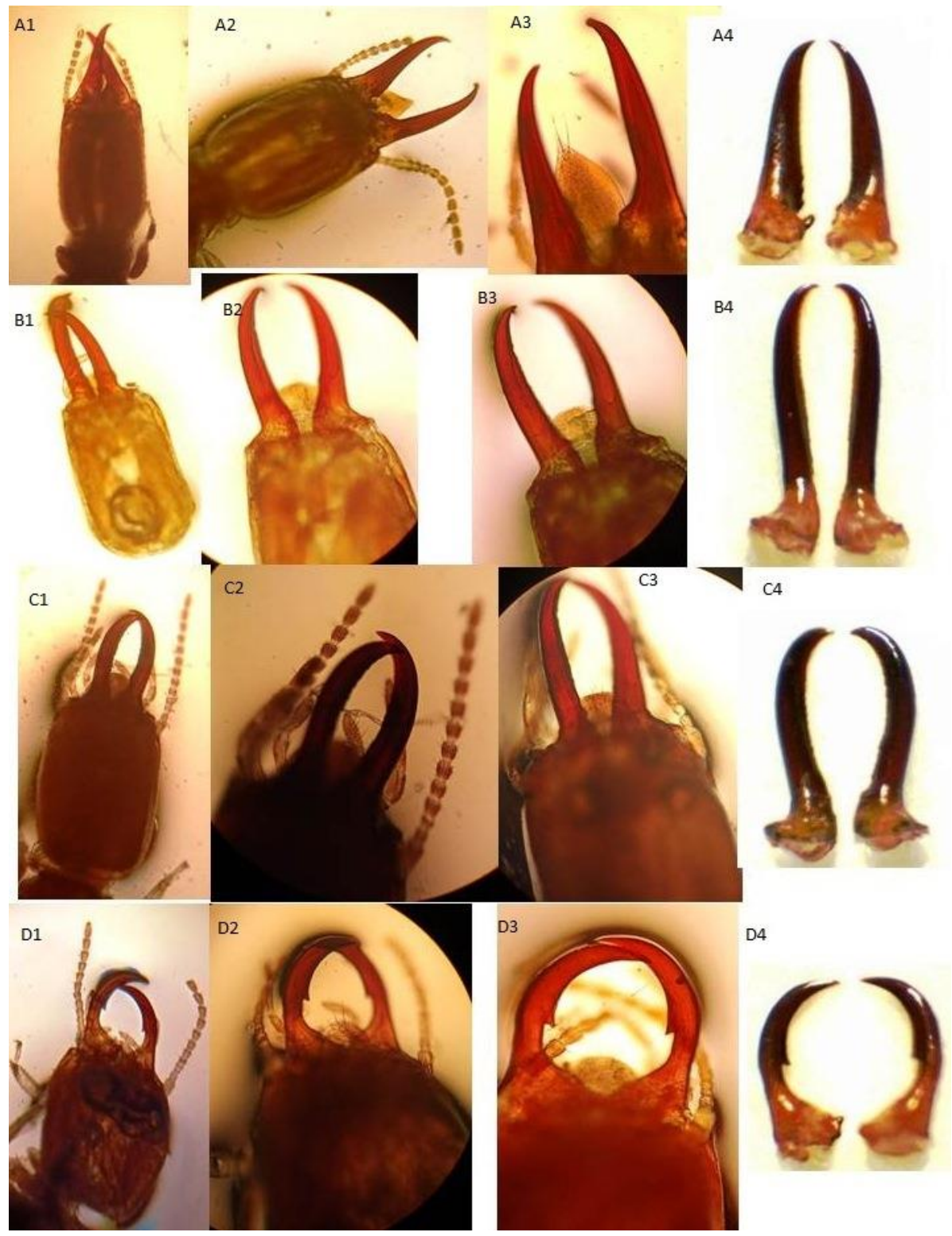

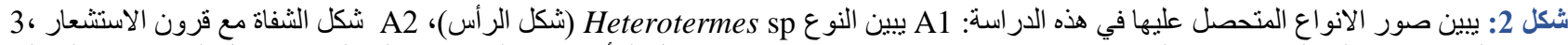

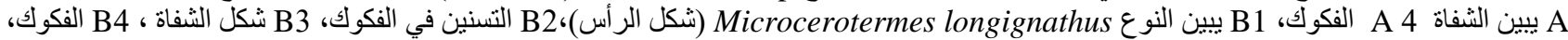

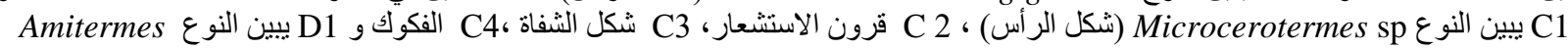
D2 شكل الثفاة ، D3 الثفاة مع الفكوك ، D4 شكل الفكوك. 
[15] S. A. Ba-Angood, Termite problems in Tihama with particular reference to oil seed crops and recommendation for control strategies AOAD, UNDP and AREA, 45pp, 1994.

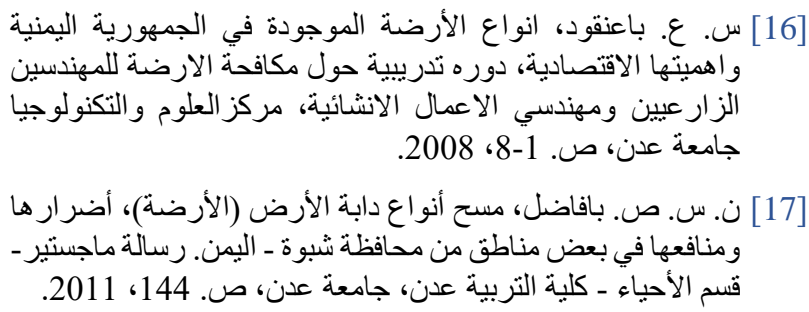

[18] W. V. Harris, Isoptera - Exped. S-W. Arabia 1937-8, London, British Museum (Nat. Hist), no. 1, pp. 421 433, 1957.

[19] R. H. Cowie, Report on second visit to people's Democratic Republic of Yemen to asses termite damage to crops. 7-12 September, Overseas Development Natural Resources Institue, London, U.K, 19pp, 1988.

[20] R. H. Cowie, Report on a visit to people's Democratic Republic of Yemen to asses termite damage to crops. 7-21 September, Overseas Development Natural Resources Institute, 29pp, 1987.

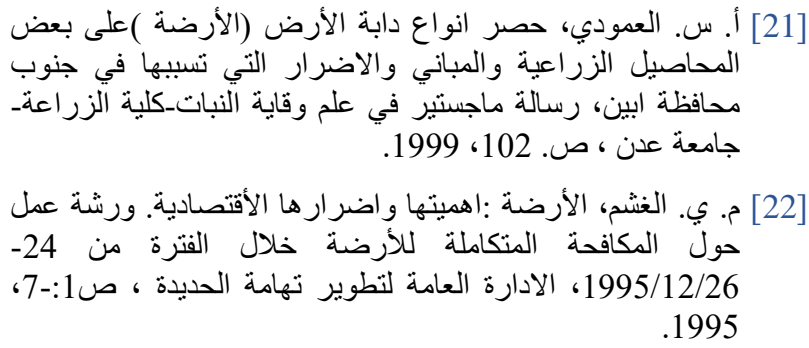

ORCID (iD)

عبدالله ناصر الهندي: 0124-0000-0002-0027
[1] م. ي. الغثم، الأهمية الأقتصادية لحشرة الأرضة الدورة التدرييية

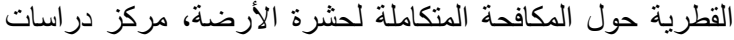

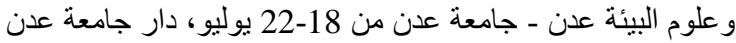

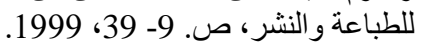

[2] P. S. Ngee, Wood resistance and wood preference, and development of suitable bait matrix for the control of subterranean termites in Malaysia. MSc. Thesis University Sains Malaysia, 221pp, 2003.

[3] S. Kambhampati and P. Eggleton, Taxonomy and phylogeny of termites. Termites: Evolution, sociality, symbioses, Ecology. Kluwer Academic Publishers, 23pp, 2000.

[4] A. Yusuf, Termite raiding by the ponerine and Pachycondyla analis (Chymenoptera:fromicidae) Behavioural and chemical Ecology. Chapter 1in: A $\mathrm{PhD}$ Thesis -University of Pretoria- Pretoria), pp. 26-49, 2010

[5] V. Lewis, UNEP/FAO/Global Facility Workshop Termite Biology and Management Feb. 1-3, 2000 Geneva, Switzerland. Chairman's Report. 12pp, 2000.

[6] R. V. Varma, "The termite problem in forest plantations and its control in India.Sociobiology", Vol. 17:155-166, 1990.

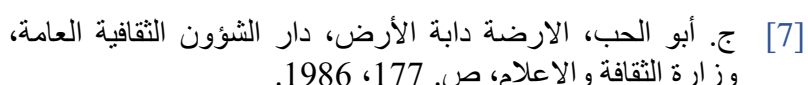

[8] R. H. Cowie, The zoogeogra physical composition and distribution of the Arabian termite fauna. Biological Jourrnal of the Linnean Society, Vol. 36, pp. 157-168, 1989.

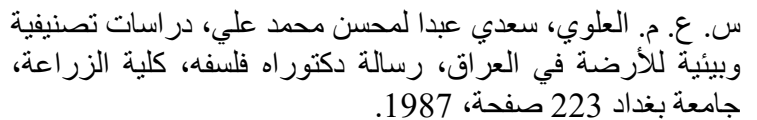

[10] O. B. Chhotani and G. Bose, Isoptera of Saudi Arabia (part 4). Fauna of Saudi Arabia, Vol. 7, pp. 122-124, 1985.

[11] خ. م. س. الغامدي، دراسة حقلية على أعشاش النمل الأبيض، الهئية

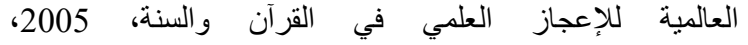
.http//www.hooran.arg/0/9/9.8.htm

[12] W. Kaakeh, "Identification, Geographical Distribution and Hosts of Subterranean Termites in the United Arab Emirates Arid Ecosystem.” Journal of Agricultural and Marine Sciences [JAMS], vol. 10 , no. 1 , pp. $33-39,2005$,

DOI: 10.24200/jams.vol10iss1pp33-40.

[13] M. Bednarzilk, Termites as plant pests in the Arab Republic of Yemen Distribultion, Damage and possible Methods of control. Intstitue of Plant Diseases, University of Bonn, 1983.

[14] W. S. Ahmed, Report on practical work carried out during a training course on the taxonomy, biology, ecology and control of termites, Natural Resources Institute UK, 122pp, 1994. 


\section{RESEARCH ARTICLE}

\section{A SURVEY STUDY OF TERMITES IN ADEN GOVERNORATE - YEMEN}

\section{Nasser S. Ba-Fadel ${ }^{1}$, Saeed A. Ba-Angood ${ }^{2}$, and Abdullah N. Al-Hindi ${ }^{3}$ * (i)}

${ }^{1}$ Biology department, Shabwah education, Aden University

${ }^{2}$ Department of Plant Protection, Faculty of Agriculture, Aden University

${ }^{3}$ Biology department, Aden education, Aden University

*Corresponding author: Abdullah Al-Hindi; E-mail: abalhindi2007@yahoo.com

Received: 01 August 2020 / Accepted: 24 October 2020 / Published online: 26 December 2020

\section{Abstract}

Termites are distributed in the tropical and semitropical areas which include the Arabian Peninsula. Thirty-three species of termites were recorded in the Arabian Peninsula especially in the southwestern of Arabia which is related to the fauna of tropical Africa (Ethiopian region). This study aims to record the termite species in different habitats in Aden governorate. The samples of the termites collected by broken the dry trees, subway on the wall, covers of the old houses and windows and wood doors from five different regions from Aden during the period of November 2016 to November 2017. The result reveals four species of termites, which are: Heterotermes sp., Microcerotermes $\mathrm{sp} 1$, Microcerotermes longignathus and Amitermes messinae (Fuller, 1922) which related to three genera that belong to two families: Termitidae and Rhinotermitidae. The species Microcerotermes longignathus is the first record in Aden governorate, While Heterotermes sp is considered the most widespread in Aden governorate with $64 \%$.

Keywords: Termites, Distribution, Species, Aden, Yemen.

ن. س بافاضل، س. ع. ا باعنقود، و ع. ن الهندي، "در اسة حصرية لأنو اع الارضة في محافظة عدن - اليمن"، مجلّة جامعة عدن الإكترونيّة للعلوم الأساسيّة

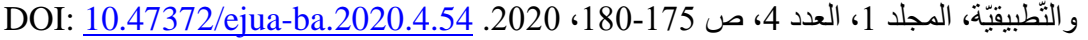

حقوق النشـر C) 2020 من قبل المؤلفين. المرخص لها EJUA، عدن، اليمن. هذه المقالة عبارة عن مقال مفتوح الوصـول يتم نوزيعه

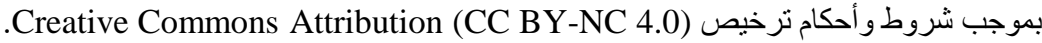

